\title{
ERGONOMIA 4.0 COMO SOLUÇÃO PARA O ABSENTEÍSMO E PARA PREVENÇÃO DE LESÕES POR ESFORÇOS REPETITIVOS E DISTÚRBIOS ÓSTEOMUSCULARES NO TRABALHO.
}

\author{
F. A. C. Silva ${ }^{1, *}$; R. R. Santos ${ }^{1}$; R. A. Moura ${ }^{1}$ \\ 1 Faculdade de Tecnologia de São José dos Campos - Professor Jessen Vidal \\ Av. Cesare Mansueto Giulio Lattes, 1350 - Eugênio de Melo, São José dos Campos/SP, \\ CEP: 12247-014, Brasil. \\ Telefone: (12) 3905-2423 \\ *fcouti@gmail.com
}

\begin{abstract}
RESUMO: A indústria 4.0 ou fábrica inteligente requer novos postos de trabalho, decretando o fim da mão de obra não especializada e recepcionando os especialistas que programarão e manusearão as máquinas, as atualizando tecnologicamente com dados precisos e confiáveis, como por exemplo, reduzir e eliminar os impactos que causam danos na saúde e à segurança ocupacional humana. Este trabalho objetiva utilizar tecnologias, métodos e processos para atender o conceito 4.0 de fabricação, melhorando as condições de trabalho, produtividade e conforto dos empregados. O método será através de inovações em um posto de trabalho com bancadas inteligentes e funcionais, como, soluções de conforto, produtividade e qualidade para redução do absenteísmo na indústria. O resultado serão princípios da manufatura enxuta, com demonstração e validação dos benefícios que impactam na redução dos níveis de absenteísmo e consequentemente do orçamento fabril.
\end{abstract}

PALAVRAS-CHAVE: Indústria 4.0; Ergonomia; Absenteísmo; Conforto dos empregados.

ERGONOMICS 4.0 AS A SOLUTION FOR ABSENTEISM AND PREVENTION OF INJURIES FOR REPETITIVE EFFORTS AND OSTEOMUSCLES DISORDERS AT WORK.

ABSTRACT: Industry 4.0 or intelligent factory requires new jobs, decreeing the end of the unskilled labor and welcoming specialists who will program and handle the machines, updating them technologically with accurate and reliable data, such as reduce and eliminate impacts on human health and occupational safety. This work aims to use of technologies, methods and processes to meet the 4.0 manufacturing concept, improving the working conditions, productivity and comfort of employees. The method will be through innovations in a workplace with intelligent and functional benches, such as comfort, productivity and quality solutions to reduce absenteeism in the industry. The result will be principles of lean manufacturing, with demonstration and validation of the benefits that affect the reduction of absenteeism levels and consequently of the manufacturing budget.

KEYWORDS: Industry 4.0; Ergonomics; Absenteeism; Comfort of Employees.

\section{INTRODUÇÃO}

A Indústria 4.0 ou fábrica inteligente contará com robôs e interfaces de alto desempenho para automatizar processos embasados em dados, reabrindo a discussão sobre o papel do ser humano 
nesta cadeia fabril, ensejando questões como, se o ser humano perderá seu emprego para máquina frente às novas tecnologias (SCHWAB, 2016).

Embora a inteligência seja artificial, novos postos de trabalho e cargos serão necessários, decretando talvez o fim da mão de obra não especializada e recepcionando os especialistas que programarão, cuidarão e manusearão as máquinas com atualizações constantes na área tecnológica com informações precisas, confiáveis e consistentes, como por exemplo, reduzir, substituir ou eliminar os impactos favoráveis que causam danos na saúde e à segurança ocupacional dos seres humanos (MAENO et al., 2006; SLACK et al., 2013).

O conceito da indústria 4.0 surgiu na Alemanha para manter o país entre os líderes mundiais continuará com mais eficiência e não se limitará somente às empresas, pois o conceito mais evidente dessa revolução é a digitalização das informações frente às demandas em pesquisas e desenvolvimento que oferecerão oportunidades para profissionais tecnicamente capacitados e formação multidisciplinar na compreensão e trabalho com a tecnologia que compõe uma fábrica inteligente (SCHWAB, 2016).

Segundo o jornal Valor, o governo federal brasileiro investiu recentemente mais de $\mathrm{R} \$ 8,6$ bilhões no fórum econômico mundial para incentivar a modernização das fábricas como forma de estimulação da Indústria 4.0, partindo do financiamento e ajuda na importação de robôs. Contudo o Brasil ainda não aproveitará todas as possibilidades de aumentar a produtividade e eficiência das empresas devido a organização do posto de trabalho e sua infraestrutura (VALOR, 2018).

Nesse viés, ocorrem os afastamentos por problemas de saúde ligados diretamente ao tipo ou à qualidade do ambiente de trabalho, ora por lesões por esforços repetitivos (LER) ora por distúrbios osteomusculares relativos ao trabalho (DORT), com crescente impacto (Figura 1) sobre a saúde do trabalhador brasileiro no anuário do sistema público de emprego e renda do Dieese, feito a partir da relação anual de informações sociais do ministério do trabalho e emprego (DIEESE, 2014).

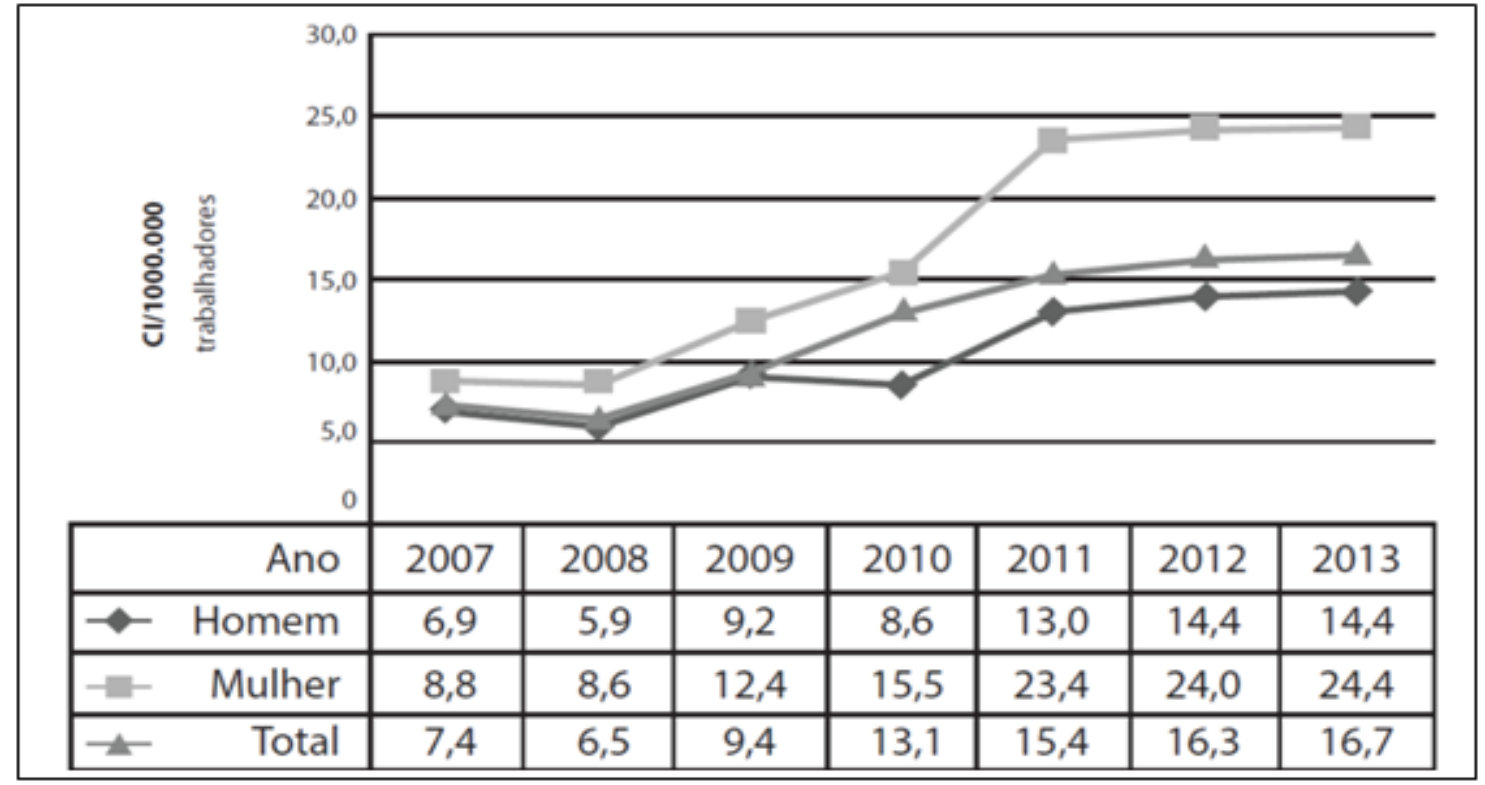

Figura 1. Estatística com níveis de afastamentos na indústria por LER/DORT.

Neste gráfico na prática, o resultado é o absenteísmo, pois as limitações das atividades diárias causadas pela LER ou DORT impactam no comportamento e atitudes como dificuldades em comparecer ao posto de trabalho, necessidade de tratamentos terapêuticos e de reabilitação, observando que com toda sua eficiência e eficácia o tratamento é corretivo e não mais preventivo (IBGE, 2013; SINAN, 2016). 


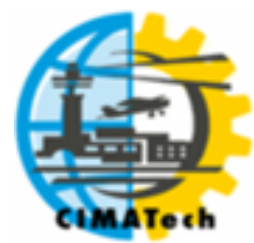

Segundo Chiavegato e Pereira (2004), estas doenças compreendem uma heterogeneidade de distúrbios funcionais e/ou orgânicos, com sintomas como fadiga muscular, dor, parestesia (sensação desagradável na pele como queimação e coceira), sensação de peso, mal estar e processos inflamatórios em tendões e ligamentos.

Além disso, os trabalhadores adoecidos ou acometidos por lesões temporárias ou permanentes, por não conseguirem se reinserir no mercado de trabalho e sentirem a dor da incapacidade, buscam ajuizar ações na justiça do trabalho, na expectativa de serem reintegrados ou indenizados (KURIONKA et al., 2005).

Ressalta-se que em 2007 foi iniciada a implantação das notificações com os conteúdos da Saúde do Trabalhador no SINAN (sistema de informação de agravos de notificação), especialmente após a rede nacional de atenção integral à saúde do trabalhador (RENAST) investir em programas de treinamento, com cursos preparatórios das equipes das unidades sentinelas, serviços de saúde com a responsabilidade notificar e divulgar os afastamentos e agravos específicos (GALDINO et al., 2012), conforme Figura 1.

O resultado prático de não se observar o conforto e posto de trabalho dos trabalhadores é o absenteísmo, pois as limitações das atividades diárias causadas pela LER ou DORT impactam nos momentos interturnos dos trabalhadores, como dificuldades em comparecer ao posto de trabalho, realizar afazeres do cotidiano (IIDA, 2005).

, Em virtude disso, o boletim da PNS investigou sobre processos terapêuticos e de reabilitação, observando que $25,4 \%$ dos entrevistados realizaram algum tipo de exercício e/ou fisioterapia para minimizar os efeitos da LER/DORT e destes 35\% usaram ou fizeram uso de tratamento com injeções ou medicamentos.

Segundo Welch (2018), quando o ritmo da mudança dentro da empresa for ultrapassado pelo ritmo da mudança fora dela, o fim está próximo, e a indústria 4.0, tema do momento, precisa se harmonizar com as questões ergonômicas, haja vista que, na mensagem do grande guru, uma empresa de sucesso precisa ter ambientes de trabalhos adequados aos seus clientes internos e estes aos executarem suas tarefas complexas garantirão o atendimento às expectativas e satisfação dos clientes externos.

Portanto, este trabalho objetiva utilizar tecnologias, métodos e processos para o desenvolvimento de novos produtos confeccionados a partir do conceito 4.0 de fabricação e prestação de serviços para empresas locais e seus fornecedores (BRASIL, 2013) apresentando inovações na organização de um posto de trabalho, criando uma bancada "conceitual" para reduzir/eliminar riscos ergonômicos e desperdícios provenientes das atividades de montagens e/ou serviços através de uma avaliação postural e comportamental durante a realização das atividades.

\section{MATERIAIS E MÉTODOS.}

Este trabalho trata-se de um artigo tecnológico, pois traz uma contribuição para a disseminação da aplicação de técnicas na solução de problemas na área técnica e científica, adotando a hierarquia da eliminação de riscos para garantir um posto de trabalho sob a premissa saúde e higiene ocupacional, com desenvolvimento e processamento do posto de trabalho conceitual e protótipos da bancada que programe movimentos ergonomicamente corretos e produtividade programada (Figura 2). 


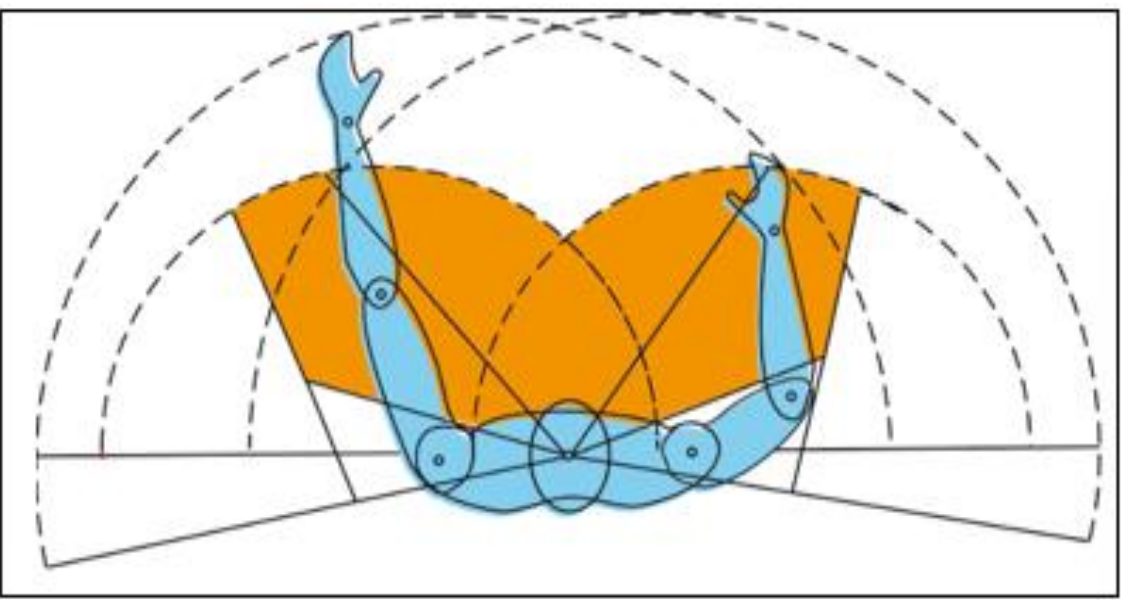

Figura 2. Estudo dos movimentos e limitações ergonômicas.

O estudo dos movimentos programados permite desenvolver a função com biomecânica ocupacional balanceada e bem definida, através de um planejamento e trabalhos com cartas de controle padronizadas e sensorização irá evitar excesso de movimentos desnecessários e improdutivos (Figura 3).

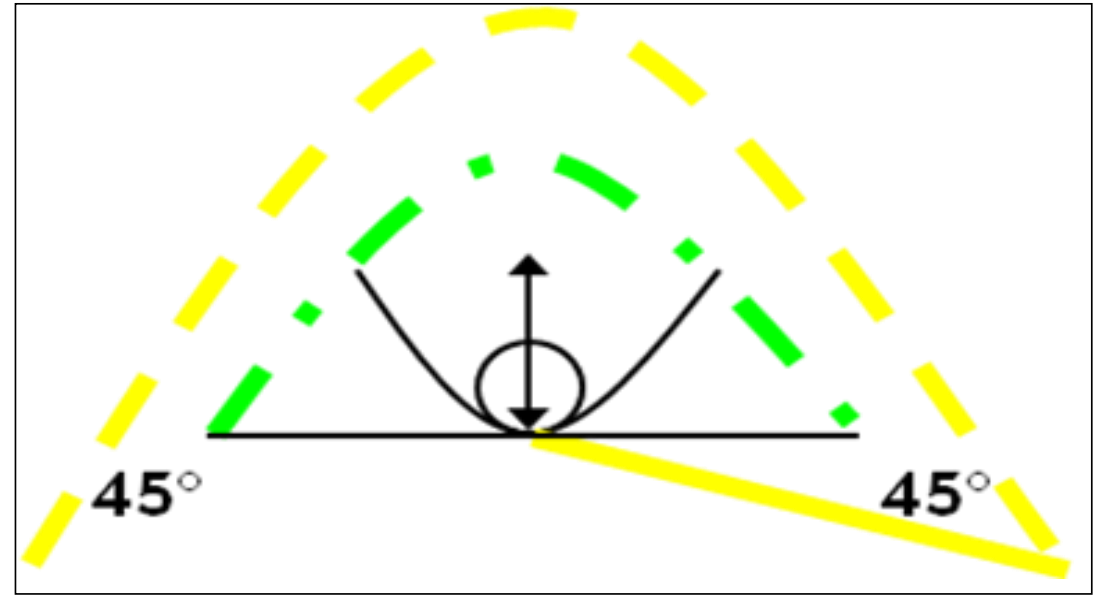

Figura 3. Movimentos sem torções e alcance do montador.

\section{RESULTADOS E DISCUSSÃO}

A parceria entre empresas e instituições acadêmicas, para a pesquisa e desenvolvimento de métodos e produtos que apliquem conceitos ergonômicos e de produtividade, demonstrou sensível redução no desconforto em certas operações fabris e consequente redução dos níveis de absenteísmo que pode ser apurado no fechamento do ano fabril de 2018, quando a bancada conceitual, adotada durante o segundo semestre de 2018 e absorvida pelo takt time da operação, deverá demonstrar a diferença.

Depois de observado o número de registros de LER/ DORT na indústria brasileira, pode-se fazer uma análise da saúde ocupacional, ergonomia e antropometria com identificação, reconhecimento, registro e entendimento da LER/DORT e sua causa raiz. 


\section{CONCLUSÃO}

Uma vez reconhecido o problema, tornam-se possíveis as medidas de prevenção e garantia de condições e organização do posto de trabalho que preservem a integridade física e psicológica dos trabalhadores, considerando as especificidades da atividade.

Os níveis de absenteísmos por LER/DORT, podem ser reduzidos com planejamento de ações de atenção integral à saúde dos trabalhadores, quantificando e qualificando a atuação de todos profissionais de saúde e proporcionando o estabelecimento de uma relação positiva entre o trabalho e a saúde, organizando o posto de trabalho com dispositivos, como bancadas, e aprimorando os comportamentos prevencionistas.

\section{AGRADECIMENTOS}

Os autores agradecem à Fatec Professor Jessen Vidal, que além do ambiente educativo, criativo e amigável, proporcionou através do V CIMATech, evento internacional, a oportunidade da publicação deste artigo, como também agradecemos a todos os envolvidos que contribuíram e contribuem direta ou indiretamente para a nossa carreira acadêmica.

\section{REFERÊNCIAS}

BRASIL. Ministério da Previdência Social. Anuário estatístico da previdência social 2013. Brasília, DF; 2014 [citado em 2015 maio 15]. Disponível em: http://bit.ly/2eK6o9X. Acesso em 23 mai. 2018.

CHIAVEGATO L. G., PEREIRA A Jr. Work related osteomuscular diseases: multifactorial etiology and explanatory models. Interface (Botucatu). 2004;8(14):149-62.

DIEESE.

Disponível em: https://cse.google.com/cse?cx=001142025415398459194\%3arsbq1_npocy\&q=afastamento\&x=0\& $\mathrm{y}=0$. Acessado em: 23 mai. 2018.

GALDINO A. G., SANTANA V. S., FERRITE S. Os centros de referência em saúde do trabalhador e a notificação de acidentes de trabalho no Brasil. Cad. Saúde Pública. 2012;28(1):145-159.

IBGE. Instituto Brasileiro de Geografia e Estatística. Pesquisa Nacional por Amostra de Domicílios: pesquisa básica - 2001 a 2014. IBGE; 2013 [citado em 2015 maio 15]. Disponível em: http://bit. ly/29nCrxm. Acessado em: 23 mai. 2018.

IIDA, I. Ergonomia: projeto e produção. 2. ed. rev. e ampl. São Paulo: Blücher, 2005.

KURIONKA I., FORCIER L., HAGBERG M., SILVERSTEIN B., WELLS R., SMITH M. J., Works related musculoskeletal disorders (WMSDs): a reference book for prevention, London: Taylors \& Francis; 2005.

MAENO M, SALERMO V, ROSSI GAD, FULLER R. Lesões por Esforços Repetitivos (LER), Distúrbios Osteomusculares Relacionados ao Trabalho (DORT), Dor relacionada ao trabalho: Protocolos de atenção integral à Saúde do Trabalhador de Complexidade Diferenciada. Brasília, DF: Ministério da Saúde, 2006. 
SCHWAB, K. A quarta revolução industrial. ISBN: 9788572839785, 1ªd. Editora Edipro, 2016.

SINAN. Sistema de informação de agravos de notificação. Centro colaborador da vigilância aos agravos à saúde do trabalhador. Sistema de Informação de Agravos de Notificação - Sinan. [citado em 2016 nov 22]. Disponível em: http://bit.ly/2gyD3ok. Acessado em 23 mai. 2018.

SLACK N.; CHAMBERS S.; JOHNSTON R.; BETTS A. Gerenciamento de Operações e de Processos. Porto Alegre: Bookmam Editora, 2013.

VALOR. Jornal. Governo federal brasileiro investiu mais de R \$ 8,6 bilhões no fórum econômico mundial para incentivar a modernização das fábricas como forma de estimulação da Indústria 4.0. Disponível em: http://www.valor.com.br/brasil/5383707/industria-40-tera-credito-de-r-86-bi. Acessado em 23 mai.2018.

WELCH, J. Empreendedorismo, As 20 Lições de Jack Welch. Disponível em: https://blog.egestor.com.br/jack-welch/>. Acessado em 23mai. 2018. 